Case Report

\title{
Nocardia transvalensis Disseminated Infection in an Immunocompromised Patient with Idiopathic Thrombocytopenic Purpura
}

\author{
Jorge García-Méndez, ${ }^{1,2}$ Erika M. Carrillo-Casas, ${ }^{3}$ \\ Andrea Rangel-Cordero, ${ }^{4}$ Margarita Leyva-Leyva, ${ }^{3}$ Juan Xicohtencatl-Cortes, ${ }^{5}$ \\ Roberto Arenas, ${ }^{6}$ and Rigoberto Hernández-Castro ${ }^{7}$ \\ ${ }^{1}$ Departamento de Posgrado y Educación Médica Continua, Instituto Nacional de Cancerología, Mexico \\ ${ }^{2}$ Departamento de Microbiología, Facultad de Medicina, UNAM, 04510 Coyoacán, MEX, Mexico \\ ${ }^{3}$ Departamento de Biología Molecular e Histocompatibilidad, Dirección de Investigación, \\ Hospital General "Dr. Manuel Gea González", 14080 Tlalpan, MEX, Mexico \\ ${ }^{4}$ Laboratorio de Microbiología Clínica, Instituto Nacional de Ciencias Médicas y Nutrición "Salvador Zubirán”, \\ 14080 Tlalpan, MEX, Mexico \\ ${ }^{5}$ Departamento de Infectología, Hospital Infantil de México "Federico Gómez”, Dr. Márquez 162, Cuauhtémoc, \\ 06720 Ciudad de México, DF, Mexico \\ ${ }^{6}$ Servicio de Micología, Hospital General "Dr. Manuel Gea González", 14080 Tlalpan, MEX, Mexico \\ ${ }^{7}$ Departamento de Ecología de Agentes Patógenos, Hospital General “Dr. Manuel Gea González”, 14080 Tlalpan, MEX, Mexico
}

Correspondence should be addressed to Rigoberto Hernández-Castro; rigo37@gmail.com

Received 24 February 2016; Accepted 4 April 2016

Academic Editor: Larry M. Bush

Copyright ( 2016 Jorge García-Méndez et al. This is an open access article distributed under the Creative Commons Attribution License, which permits unrestricted use, distribution, and reproduction in any medium, provided the original work is properly cited.

Nocardia transvalensis complex includes a wide range of microorganisms with specific antimicrobial resistance patterns. $N$. transvalensis is an unusual Nocardia species. However, it must be differentiated due to its natural resistance to aminoglycosides while other Nocardia species are susceptible. The present report describes a Nocardia species involved in an uncommon clinical case of a patient with idiopathic thrombocytopenic purpura and pulmonary nocardiosis. Microbiological and molecular techniques based on the sequencing of the 16S rRNA gene allowed diagnosis of Nocardia transvalensis sensu stricto. The successful treatment was based on trimethoprim-sulfamethoxazole and other drugs. We conclude that molecular identification of Nocardia species is a valuable technique to guide good treatment and prognosis and recommend its use for daily bases diagnosis.

\section{Introduction}

Nocardia species are Gram-positive ubiquitous, aerobic actinomycetes, saprophytic of soil, water, and organic matter Nocardia genus is an opportunistic pathogen which may cause disease in immunocompromised or immunocompetent patients $[1,2]$. The primary source of infection is through inhalation. However, more than $90 \%$ patients have underlying conditions compromising their cellular or humoral immunity. $N$. transvalensis must be differentiated due to its natural resistance to aminoglycosides, while other Nocardia species are typically susceptible such as $N$. blacklockiae and N. wallacei $[3,4]$.

N. transvalensis was first described by Pijper and Pullinger in 1927 as the causative agent of mycetoma of the foot in a South African patient [5]; since then, it has turned to be a cause of life threatening infections and other nonthreatening infections [4]. N. asteroides and N. brasiliensis are common species recognized in clinical cases, while $N$. transvalensis sensu stricto is one of the least frequent Nocardia species. It has restricted susceptibility to cotrimoxazole, third-generation cephalosporin, imipenem, and linezolid. $N$. 
transvalensis is a pathogen rarely reported, particularly in a case of cystic fibrosis with chronic pulmonary infections [5], brain abscess [3], keratitis [4], ocular infections, HIV patients $[6]$, mycetoma [1,7], and pulmonary infections [8].

Here we present an uncommon report of pulmonary nocardiosis with haematological and neurological involvement caused by $N$. transvalensis in a patient with idiopathic thrombocytopenic purpura.

\section{Case Description}

A 59-year-old male from Guerrero, Mexico, was referred to the National Cancer Institute at Mexico City, diagnosed with prostate adenocarcinoma (Gleason 8), and treated with flutamide (Androgen Receptor Inhibitor) plus goserelin (gonadotropin releasing hormone superagonist (GnRH agonist)). After exhaustive physical examination and thrombocytopenia $\left(51 / \mathrm{mm}^{3}\right)$, presumptive drug-induced idiopathic thrombocytopenic purpura was recognized. This condition was managed with prednisone $100 \mathrm{mg}$ /day plus 6mercaptopurine $50 \mathrm{mg} /$ day, for 7 weeks. After 12 weeks at his hometown, the patient developed progressive respiratory insufficiency, diagnosed with right pneumonia and treated with oral levofloxacin (500 mg/daily). He was readmitted to the Cancer Institute with hyperglycaemia because of steroid use, and no bacterial isolation was obtained from the first sputum. One week later, the patient was received at the Emergency Room complaining of fever $\left(39^{\circ} \mathrm{C}\right)$, dyspnea, cachexia, and haemoptysis. Clinically with right basal hypoventilation, the imaging studies (chest X-ray, followed by a thoracic computed tomography scan) revealed cavitated right middle lobe pneumonia, as well as lesions in other lung segments (Figures 1 and 2). The antimicrobial regimen was changed to ceftriaxone and clindamycin. In spite of mild improvement, the patient was submitted to a CT-guided lung biopsy. Gram and Ziehl-Neelsen stains were negative and until the fourth day waxy colonies were detected in blood agar and Sabouraud plates under incubation at $37^{\circ} \mathrm{C}$ and $5 \% \mathrm{CO}_{2}$ atmosphere. Microscopically, weak Gram-positive, branched filamentous bacilli were observed. Trimethoprim-sulfamethoxazole $(15 \mathrm{mg} / \mathrm{kg} /$ day) was the successful treatment. Almost immediately to diagnosis, the patient referred to right arm weakness, which reversed on the third day of treatment with trimethoprim-sulfamethoxazole. A Magnetic Resonance of the brain reported minimum changes in the meningeal layers, and the lumbar puncture showed moderate pleocytosis without recovering any microorganisms. A follow-up thoracic CT scan showed the resolution of more than half of lung involvement and trimethoprim-sulfamethoxazole was continued at home. The resolution of the idiopathic thrombocytopenic purpura was followed by tapering prednisone and 6 -mercaptopurine $(25 \mathrm{mg} / 48 \mathrm{~h})$ for the next 6 and 12 months until discontinuation. One year after the episode and maintenance treatment with amoxicillin-clavulanate (40 mg/10 mg/kg/day) plus trimethoprim-sulfamethoxazole (15 mg/kg/day) for the Nocardia infection, the imaging studies confirmed the complete resolution of the lung pathology. Since then, the patient has not relapsed and the cancer treatment continued.

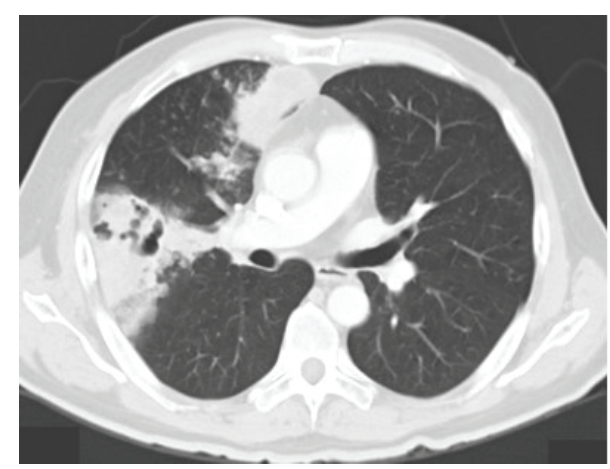

FIGURE 1: Axial computed tomography showing middle right lobe involvement.

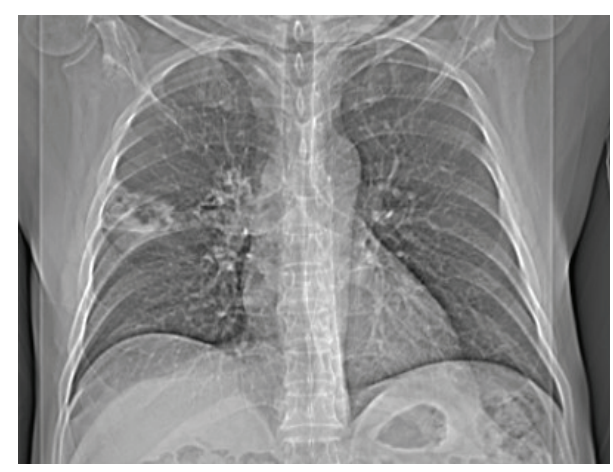

FIgURE 2: Chest computed tomographic scan at ER admission.

\section{Materials and Methods}

The genus identification was based on the Gram-positive stain of branching and filamentous bacilli, positive modified acid-fast stain, colonial morphology, and conventional biochemical reactions. Further species identification was based on sequencing of the $16 \mathrm{~S}$ rRNA gene, using the primers Noc1 (5'-GCTTAACACATGCAAGTCG-3') and Noc2 $\left(5^{\prime}\right.$ GAATTCCAGTCTCCCCTG-3') [9]. The PCR product was purified with the QIAquick purification kit (Qiagen, Ventura, CA, USA), according to the manufacturer, and DNA sequences were determined with Taq FS Dye Terminator Cycle Sequencing Fluorescence-Based Sequencing and analysed on an Applied Biosystems 3730 DNA sequencing system (Foster City, CA, USA). The sequence of the $16 \mathrm{~S}$ showed $100 \%$ homology with N. transvalensis accession number FJ516749.

\section{Discussion}

A marked increased number of human Nocardia infections have been reported worldwide since the decade of 1960 . However, $N$. transvalensis is an infrequent pathogen and probably misdiagnosed. Pulmonary nocardiosis is a mayor clinical manifestation of Nocardia species infection and may pose a challenge when distinguishing it from tuberculosis. To the best of our knowledge, only few cases had been related involving $N$. transvalensis as the primary cause of acute or 
chronic infections. Predisposing factors of human nocardiosis are chronic obstructive pulmonary disease, bronchiectasis, pulmonary fibrosis, emphysema, asthma, neoplastic disease (thoracic or induced by potent immunosuppressant drugs), organ transplant (bone marrow or solid transplantation), or immunosuppressive therapy used in autoimmune diseases, cancer, diabetes mellitus, previous or concurrent tuberculosis, and advanced HIV infection (acquired immunodeficiency syndrome) [8].

The geographical differences among continents should be taken into account. $N$. transvalensis sensu stricto is common in Africa and absent in Asia. N. wallacei is the most frequent species isolated in the United States, whereas scarce information is available in the rest of America, maybe due to misdiagnosis or underestimation of human cases and difficulties in clinical and laboratory diagnosis.

The routine diagnosis has been microbiological culture and morphological features. However, the phenotypic identification has $37 \%$ misidentification of Nocardia species based on the 16S rRNA gene which discern among the $N$. transvalensis cluster and other species by two base insertions. Few reports have used molecular techniques to identify the involvement of $N$. transvalensis in clinical cases $[4,10]$; nonetheless, its molecular identification is of great value as guidance for treatment choice [4]. The delay in the Nocardia species identification has clinical implications because these bacteria have been classified according to their antibiotic resistance patterns but share similar clinical manifestations; therefore, the treatment election may be hindered. In the current case, the use of trimethoprim-sulfamethoxazole (an antimicrobial of choice for nocardiosis) was the right choice in combination with other antimicrobials, which may include imipenem, amikacin, ceftriaxone, and amoxicillinclavulanate.

\section{Conclusion}

The present report describes an uncommon $N$. transvalensis infection in an immunocompromised patient with idiopathic thrombocytopenic purpura. This work underlines the value of early diagnosis by microbiological culture combined with accurate molecular identification of the Nocardia species; in addition, sulfonamide extended use and the reduction in immunosuppression are key factors for good prognosis. We encourage the use of molecular identification at the species level for further understanding of the epidemiology, taxonomy, antimicrobial susceptibility, and clinical and epidemiological aspects of nocardiosis.

\section{Competing Interests}

The authors declare that there are no competing interests regarding the publication of this paper.

\section{References}

[1] S. H. Mirza and C. Campbell, "Mycetoma caused by Nocardia transvalensis," Journal of Clinical Pathology, vol. 47, no. 1, pp. 8586, 1994.
[2] M. M. McNeil, J. M. Brown, P. R. Georghiou, A. M. Allworth, and Z. M. Blacklock, "Infections due to Nocardia transvalensis: clinical spectrum and antimicrobial therapy," Clinical Infectious Diseases, vol. 15, no. 3, pp. 453-463, 1992.

[3] R. F. Yorke and E. Rouah, "Nocardiosis with brain abscess due to an unusual species, Nocardia transvalensis," Archives of Pathology and Laboratory Medicine, vol. 127, no. 2, pp. 224-226, 2003.

[4] E. Trichet, S. Cohen-Bacrie, J. Conrath, M. Drancourt, and L. Hoffart, "Nocardia transvalensis keratitis: an emerging pathology among travelers returning from Asia," BMC Infectious Diseases, vol. 11, article 296, 2011.

[5] A. Aravantagi, K. Patra, M. Broussard, and K. Jones, "A case of Nocardia transvalensis pneumonia in a 19-year-old cystic fibrosis patient," Lung India, vol. 29, no. 3, pp. 283-285, 2012.

[6] N. Poonwan, M. Kusum, Y. Mikami et al., "Pathogenic Nocardia isolated from clinical specimens including those of AIDS patients in Thailand," European Journal of Epidemiology, vol. 11, no. 5, pp. 507-512, 1995.

[7] H. C. Gugnani, J. O. Ojukwu, and A. V. Suseelan, "Mycetoma of thumb caused by Nocardia transvalensis," Mycopathologia, vol. 80, no. 1, pp. 55-60, 1982.

[8] A. Kageyama, K. Yazawa, J. Ishikawa, K. Hotta, K. Nishimura, and Y. Mikami, "Nocardial infections in Japan from 1992 to 2001, including the first report of infection by Nocardia transvalensis," European Journal of Epidemiology, vol. 19, no. 4, pp. 383-389, 2004.

[9] C.-K. Tan, C.-C. Lai, S.-H. Lin et al., "Clinical and microbiological characteristics of Nocardiosis including those caused by emerging Nocardia species in Taiwan, 1998-2008," Clinical Microbiology and Infection, vol. 16, no. 7, pp. 966-972, 2010.

[10] W. L. Liu, C. C. Lai, W. C. Ko et al., "Clinical and microbiological characteristics of infections caused by various Nocardia species in Taiwan: A multicenter study from 1998 to 2010," European Journal of Clinical Microbiology and Infectious Diseases, vol. 30, no. 11, pp. 1341-1347, 2011. 


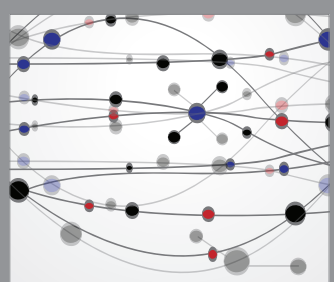

The Scientific World Journal
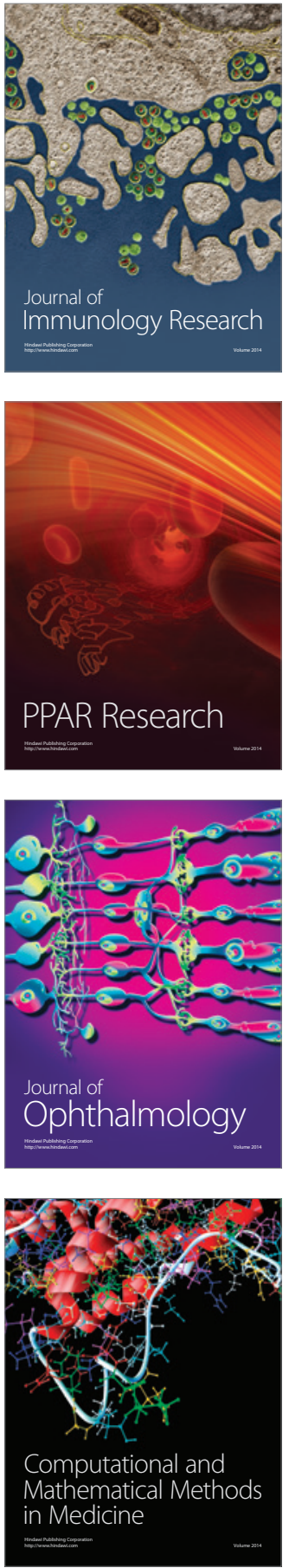

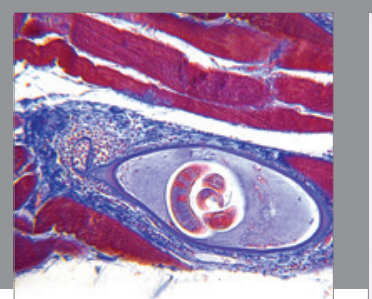

Gastroenterology Research and Practice

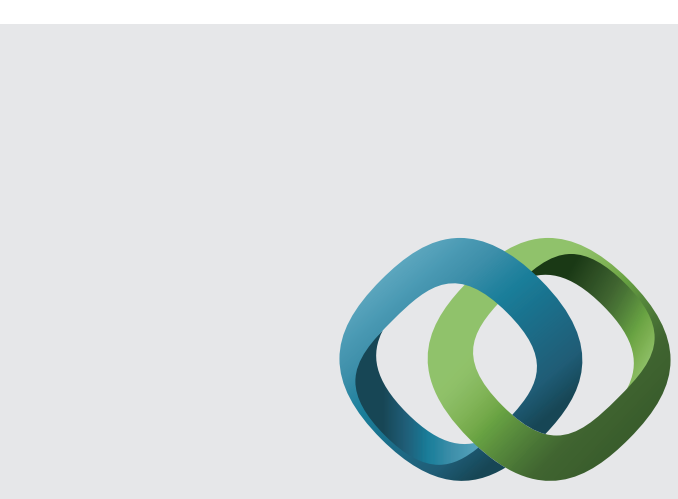

\section{Hindawi}

Submit your manuscripts at

http://www.hindawi.com
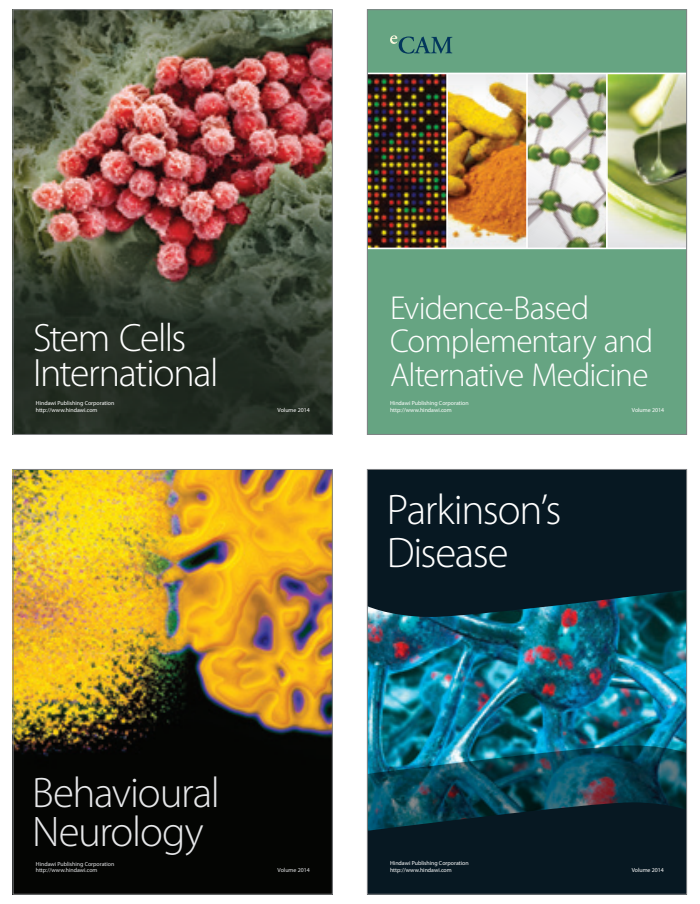
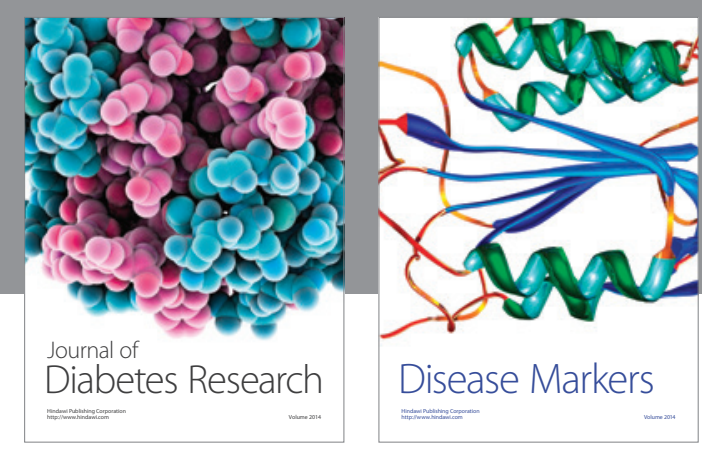

Disease Markers
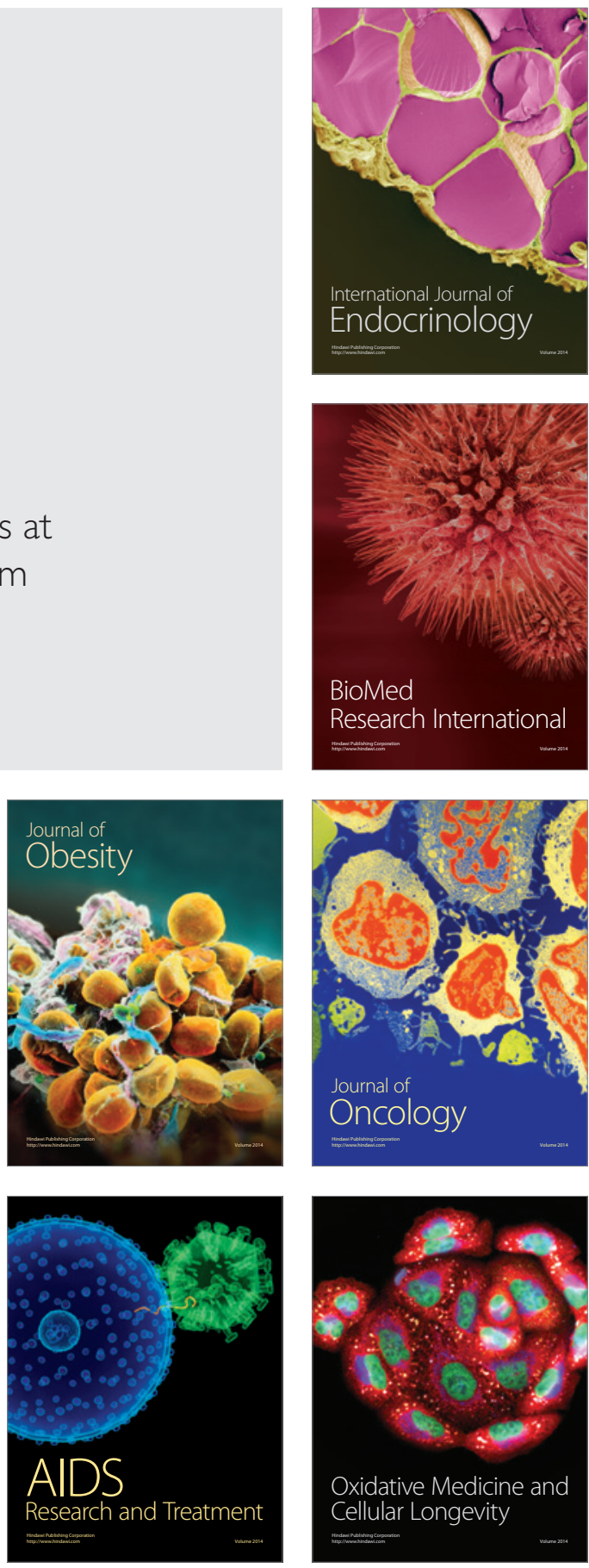